\title{
A Tunable Physical Model of Arthropod Antennae
}

\author{
Alican Demir, Edward W. Samson and Noah J. Cowan
}

\begin{abstract}
Insects rely on sensory cues-tactile, hygrometric, thermal, olfactory-gathered with a pair of head-mounted antennae to perform a wide variety of sensory guided tasks. Many questions regarding the potential impact of specific mechanical design features on antennal performance can be directly and thoroughly assessed using an artificial robotic model of an antenna. Here we describe a highly tunable, modular tactile robotic model antenna and experimentally test its tactile sensing performance using a custom testbed. Exploratory experiments demonstrate the importance mechanical "tuning" on tactile navigation performance. With this model robotic antenna, numerous mechanosensory manipulations are possible, providing a new experimental platform for future testing of specific biological hypotheses.
\end{abstract}

\section{INTRODUCTION}

Insect and arthropod antennae are small, lightweight, highly segmented, and fully integrated sensory systems that provide feedback from several modalities - touch, humidity, temperature, and olfactory. This sensory information is acquired by thousands of receptors all along the flagellum, the long, slender distal portion of the antenna. The long-term goal of this research is to understand how the passive mechanics of the antenna is coupled with the widely dispersed tactile mechanoreceptors along the length of the antenna to facilitate tactile perception in particular. However, antennae are highly complex and integrated structures that perform a wide variety of tasks, rendering it challenging to isolate and repeatedly test how specific mechanosensory design features influence tactile sensing performance.

To help overcome this gap, this paper presents a biologically inspired artificial antenna template [1] with a high level of mechanical tunability (see Figure 1). This physical model of antenna-based tactile sensing - that is, an embodied mechanical system and environmental testbed-promises to provide an essential experimental component to understand and evaluate performance given the complex interactions that occur between an antenna and its environment. Such experimental assays can be used to inform the development of mathematical models, as well as complementary but more narrowly focused experiments on the biological antennae. Such a platform can also be used to address synergistic questions regarding the necessary mechanical properties for developing task-specific high performance antennae for robotic applications.

This work was supported in part by the NSF under Grant No. 0845749 A. Demir and N. J. Cowan are with the Department of Mechanical Engineering, Johns Hopkins University, Baltimore, MD, USA, alicanejhu.edu

E. W. Samson is with Baltimore Polytechnic High School, Baltimore, MD, USA

\section{A. Motivation}

With an evolution history over 400 million years [2], arthropod antennae are arguably nature's multisensory formfactor of choice: of the estimated 5 million to 30 million animal species, more than $95 \%$ are insects [3], [4]. In fact May [5] suggests that to a rough approximation "all organisms are insects" and, consequently, we argue that to the same rough approximation all of Earth's animals have antennae. Terrific progress has been made on "vertebrateinspired" tactile sensors that mimic finger tips [6] and whiskers [7], but so far sensorized arthropod antennaedespite their unequivocal biological success-have received surprisingly little attention from the robotics community.

The biological popularity of this sensory organ [8] is not without reason. American cockroaches (Periplaneta americana), for instance, use their antennae as tactile feelers [9] and thus can rely on surfaces in the environment for tactile navigation. Even when blinded, American cockroaches execute this task so well that they can reach to speeds up to $80 \mathrm{~cm} / \mathrm{s}$ (or about 25 body lengths/s) [10], [11] (which would be analogous to a blind human running through a maze at approximately $100 \mathrm{mph}$ ). Such performance is naturally a source of inspiration for biologists and robotic system developers, but exploring the mechanics of sensing in the highly complex biological antenna is daunting. For example, Okada and Toh [12] stated as one of their early goals determining which section of the cockroach antenna (base or flagellum) is the most crucial for object orientation; they reported that preparations aimed towards isolating antennal features (such as flagellum ablation/replacement and joint immobilization) can have severe negative side effects on locomotion and behavior, thus rendering such hypothesis testing impractical; a physical robotic testbed can help fill this gap [13].

\section{B. Antennal Tactile Sensing}

For the purposes of this paper, we define a tactile antenna as a flexible, slender, possibly branching, passive tactual structure, sensorized along its length. Artificial antennal tactile sensing remains to be a relatively unexplored topic in robotics, although a few implementations have been published. These systems were designed to demonstrate a specific biological behavior, such as flow/contact discrimination [14], wall following [15], [16], [11] and obstacle clearance in the sagittal plane [17]; in all cases, the systems integrate curvature, but not contact sensing. This antenna presented in this paper takes the next step by integrating both contact and curvature sensing along the entire length, and is designed to be tunable and modular enough to facilitate experimentation 


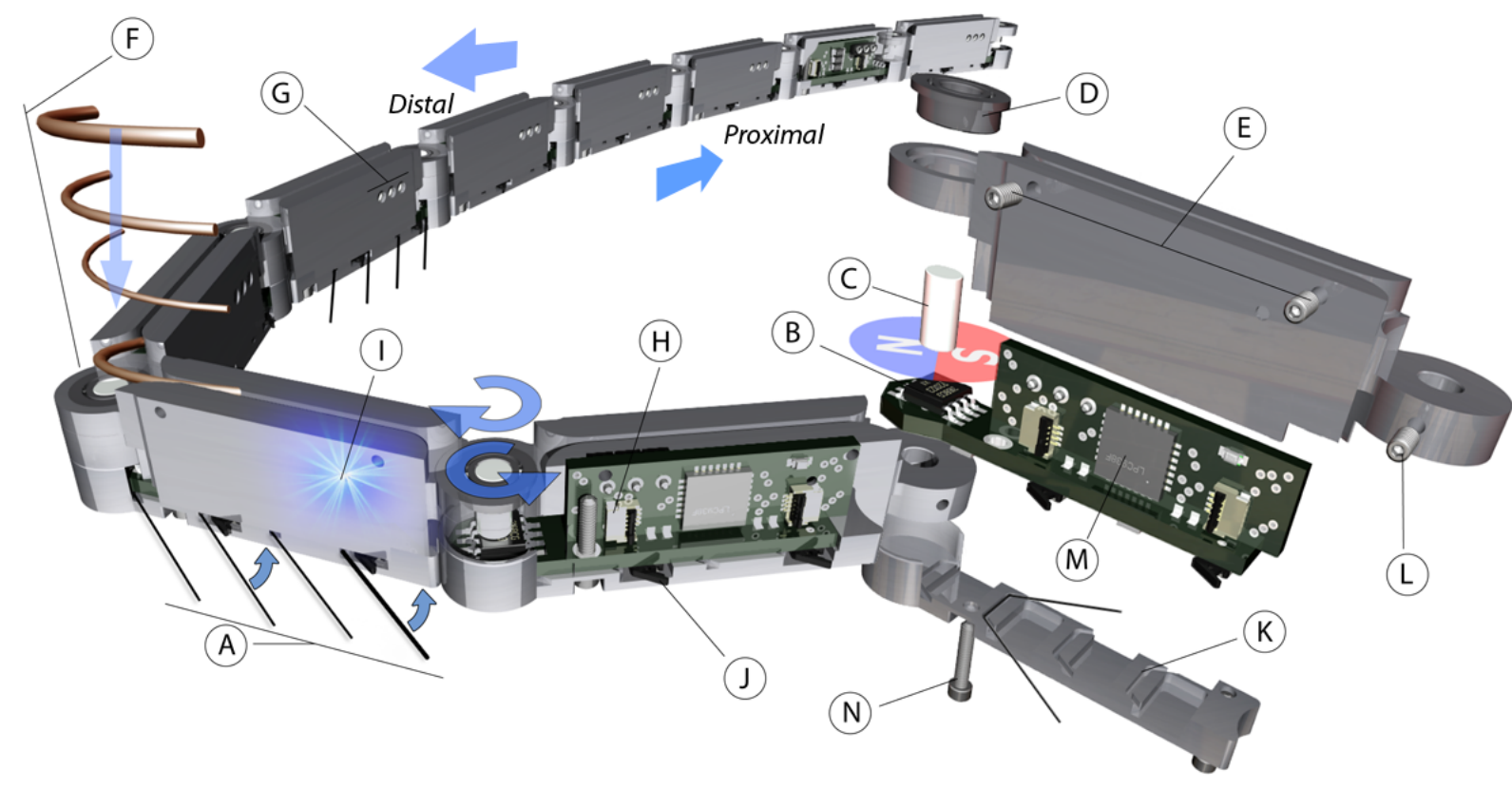

Fig. 1. Antenna components and certain features: A) Replaceable hair-like spines, B) Hall-effect sensor, C) Diametrically magnetized magnet, D) Ball-bearing, E) Set screws for holding flexible "backbone", F) Various flexible "backbone" wires, G) Firmware update port (RS-232), H) $\mathrm{I}^{2} \mathrm{C}$ and power connector, I) LED indicator, J) Detector switch, K) Spine mounting groove, L) Magnet holding set screw, M) Microcontroller, N) Mounting cap screw.

in a variety of tactile behaviors, hopefully facilitating future tests of specific biological hypotheses.

The functional capabilities of an antenna are governed both the mechanical properties, as well as the number, diversity and density of the minute sensory structures along the flagellum [18], [8]. The role of structural stiffness seems to play a significant role in antenna design in nature. For instance applying a preset force to a locust flagellum will cause different amounts of bending for different directions [19] and, in particular, the flagellum is stiffest in the direction of wind flow during forward flight. Non-uniform stiffness profiles are also utilized for example by the crayfish [14], Cherax destructor, where their flagellum consist of tapered segments [20]. This tapering differs among species but usually the function is to allow the distal tip of the antenna to deflect more easily than the proximal end. Our antenna prototype is designed to allow such mechanical variations to be incorporated easily depending on the target tactile antennal function of interest.

The biological antenna also contains many different sensilla along its flagellum. However, the thick-walled mechanosensory hairs (S. Chaetica B), which are triggered by external contacts, are of our main interest in the present work. This is due to their contribution to the tactile perception of objects but also due to their potential effects on overall mechanical behavior during sensing [21]. A typical Periplaneta americana flagellum has about 6500 such hairlike contact sensors arranged around the circumference of all segments [22], [23].

Each sensillum itself is a long, tapering, highly grooved shaft between $35 \mu \mathrm{m}$ to $250 \mu \mathrm{m}$ in length mounted in a flexible circular socket with $10 \mu \mathrm{m}$ to $25 \mu \mathrm{m}$ in diameter. The shaft of the sensillum is always slanted distally and the tip of the shaft is curved outward [23]. They are known the be the longest and most rigid hairs on the entire antenna [24]. As any contact force on the antenna should be directly propagated through those hair-like contact sensors to the main antenna support structure, it has been hypothesized by the last author and colleagues that this mechanical coupling, for example in cockroaches, is utilized to help keep the antenna in a shape favorable for wall following [21]. Specifically, if the antenna shape is not in a favorable configuration, then the distally pointing hair disposition may generate forces on the antenna that help drive it to return to the "preferred" configuration for wall following. Therefore we believe the incorporation of adjustable and replaceable hair-like spines is crucial and hence featured in our artificial antenna.

\section{Relationship to Mammalian Whiskers}

Certainly, "vertebrate-inspired" systems such as fingers and whiskers promise to provide nearly instantaneous $2 \frac{1}{2} \mathrm{D}$ information about a surface under investigation - a feat not possible with a 1D tactile sensor such as an arthropod antenna. Despite this limitation of an individual tactile antenna, arthropods readily navigate in their local environments using feedback from these 1D probes. With that in mind, it is not actually clear to what extent the 2D component of whisker data is paramount to a rat's navigational behavior per se.

Advantages of parallel spatial integration notwithstanding, individual whiskers are simply dead hairs: long slender cantilever probes from which environmental tactile properties must be estimated solely based on measurements at the base using subcutaneous receptors. Thus, an individual whisker likely transduces a tiny fraction of the tactile information 
encoded by a living arthropod antenna which boasts literally thousands of sensory structures distributed along its length.

Discerning bending and contact location using basal reception alone has been accomplished through artificial whiskers [7], [25], [26], [27]. Basal sensing may enhance tactile perception in insect antennae, but for some tactile tasks it is neither necessary nor sufficient [12], [10] and for antenna-based wall following in particular, flagellar information seems to dominate [10]. Thus, we focus here on the mechanosensory properties of the flagellum although ultimately we plan to incorporate a sensorized, actuated base.

\section{ANTENNA DESIGN}

The design is highly tunable: a user can vary the stiffness of each joint, modify (or remove) the geometric and mechanical properties the antennal hairs, increase the mass of each segment, adjust length of the antenna by adding or removing segments, and impose different antennal precurvatures.

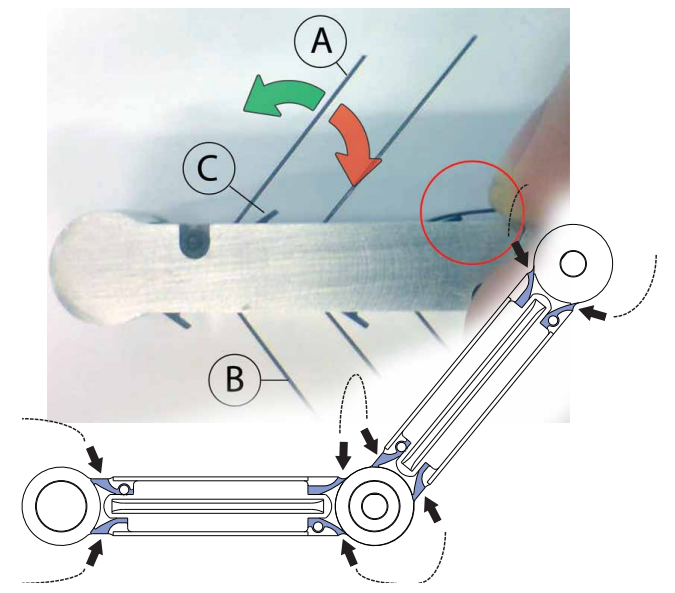

Fig. 2. TOP: Bottom view of a segment: A) Detached distally pointing active hair; B) Passive spine; C) Lever arm of the detector switch; Green Arrow: Hair bending direction will not engage the switch "C"; Red Arrow: Hair bending direction will engage the switch "C"; Red Circle: Engagement of the hair with the switch lever arm. BOTTOM: Electrical coupling between segments. Dashed lines depict possible coupling cable connections. Blue areas depict the cable path guides leading to the connectors.

As depicted in Figure 1, the antenna is a serially connected planar rigid body chain where the $40 \mathrm{~mm}$ long, modular, identical, stand-alone segments can be oriented from $90^{\circ}$ to $270^{\circ}$ relative to the adjacent segments. Each segment (Figure 2) rotates a short diametrically magnetized cylinder acting as the hinge shaft. The magnetic shaft is grasped on both axial halves, where the top half is pressfit into a stainless steel ball-bearing and the bottom half is slid into the joint hole of the distal segment chassis. A set-screw can be tightened to pinch the hinge shaft so that the magnet always turns with the distal segment. The ball-bearing allows the shaft to rotate freely with respect to the next proximal segment, where a Melexis ${ }^{1}$ Hall-effect rotary position sensor chip is positioned underneath. This Hall-effect sensor facilitates contactless absolute angular

\footnotetext{
${ }^{1}$ http: //www.melexis.com/
}

orientation measurement of the shaft's magnetic poles in $0.09^{\circ}$ resolution and outputs it as an analog voltage.

We placed four directional detector switches as binary touch sensors in the segment design, uniformly distributed along the length segment, but alternating sides (i.e. mediallateral-medial-lateral). The employed detector switch is actuated by a short distally pointing-hence directional-lever arm that flushes with the aluminum segment chassis under external contact. This ensures contact forces larger than the maximum operating force of $0.3 \mathrm{~N}$ to be properly absorbed by the chassis in order to protect the sensor (Figure 2).

The chassis for each segment is a sagittally symmetric, two piece, thin-walled, precision machined aluminum shell structure that encapsulates the entire segment circuitry when screwed together. Besides physical protection, the shell body provides four mounting grooves adjacent to contact sensors for thin hair-like extensions. The mounting grooves are oriented in a way that they are pointing towards the distal end of the antenna and parallel to the detector switch lever arms. Their operating principle is such that when a hair is bent towards the distal end of the antenna, then the lever arm of the associated contact sensor would be engaged as well. On the other hand when a hair is bent towards the proximal end of the antenna then this bending would not affect the contact sensor lever arm because of the detached nature of the hair (Figure 2).

The segment electronics subassembly is built upon two orthogonally joined PCB boards. There is a single $\mathrm{I}^{2} \mathrm{C}$ bus along with the power and ground lines that connects all segments and the host robot computer, for which $0.5 \mathrm{~mm}$ Flat Flex ribbon cable (FFC) is used between each segment. As shown on Figure 2, this coupling cable enters and leaves a segment from a side of the user's choice through special slits designed to prevent force propagation towards the connectors on the PCB during potentially aggressive antennal deflections. Details are described in [28].

\section{EXPERIMENTAL RESULTS}

\section{A. Angular Measurement Uncertainty}

We estimated the per-segment bias, $\beta_{i}$, and variance $\sigma_{i}^{2}$ as a measure of angular accuracy and precision. First, 500 joint angle measurements were sampled from a two-segment antenna configuration and repeated for five randomly selected different segments, where the segment joint angle $\phi_{i}$ is manually brought to five known reference values. Ultimately, our goal is to estimate the angle, $\phi_{i}$ of each of the joints. Neglecting quantization errors, we model our sensor as providing a measurement treated as a Gaussian random variable, with variance $\sigma_{i}$ and mean $\phi_{i}+\beta_{i}$.

The results of our tests show a varying mean accuracy $\left(\beta_{i}\right)$ ranging from $-2.33^{\circ}$ to $3.85^{\circ}$ between the extremes of $90^{\circ}$ to $270^{\circ}$, which is almost zero around the calibration angle of $180^{\circ}$. The precision is fairly good for all reference angles, which is indicated by standard error values $\left(\sigma_{i}\right)$ ranging from $0.71^{\circ}$ to $1.75^{\circ}$. We also quantified the consistency between different segment modules by the variance of 
standard errors, which varies from $0.2^{\circ}$ to $0.81^{\circ}$ for a given reference angle.

The effects of the measurement uncertainty at each joint propagate to an error in the estimate of contact location near the tip of the antenna. Assuming that an $N$ segment antenna can only sense contacts at the joints and that the segment lengths $\ell$ are identical, then the position coordinates $x_{k}, y_{k}$ of each valid contact location $k$ can be expressed using forward kinematics with respect to the base joint $\left(x_{0}, y_{0}\right)$ as

$$
\left[\begin{array}{l}
x_{k} \\
y_{k}
\end{array}\right]=\sum_{i=1}^{k} l\left[\begin{array}{l}
\cos \sum_{j=1}^{i} \phi_{j} \\
\sin \sum_{j=1}^{i} \phi_{j}
\end{array}\right] .
$$

For the purposes of providing a rough estimate of the uncertainty propagation, we need a measure of uncertainty at each joint. For this purpose, we somewhat crudely lumped our estimates of the bias and variance together to get an estimate of the mean squared error (MSE). Specifically, we used the mean of the biases and the maximum of the standard errors, respectively, to compute an effective mean-squarederror MSE $=\beta^{2}+\sigma^{2}$. In our case, this course approximation to the total uncertainty of the antenna is computed as $4.14^{\circ}$.

Assuming small variations at the joints (which is clearly valid in our case), we can estimate contact point covariance using differential kinematics. If $J$ is the Jacobian of (1), then the covariance matrix of the tip can be approximated by $\Sigma=J J^{T}$. MSE. Changes in error bounds for different antenna shapes via covariance ellipses are presented in [28].

\section{B. Spatial Mapping}

Tunability is the most important design aspect of our tactile antenna template. Thus it is crucial to verify that a change of isolated parameters do in fact alter the mechanics of sensing of the artificial flagellum. Here we chose to tune the stiffness profile as we suspect that this parameter influences the overall mechanics of sensing the most. A testbed was designed and built to facilitate repetitive spatial mapping trials with different antenna parameters.

Our testbed (Figure 4) is a $1.0 \mathrm{~m} \times 1.5 \mathrm{~m}$ aluminum frame, holding two MDF boards across. The first serves as an immobile mounting platform for the belt-driven Velmex ${ }^{2}$ computer controlled linear actuator with the antenna system, and the second as a $0 \mathrm{~m}$ to $0.6 \mathrm{~m}$ slidable platform for the "programmable" testing surface. The surface consists of 400 stacked $3.2 \times 150 \times 50 \mathrm{~mm}$ sheets of acrylic, each with a $100 \mathrm{~mm}$ long slot. A steel threaded rod traverses across the slots to held the stack together and the desired wall shape is formed by moving in or out the individual sheets. The wall's shape can be fixed by compressing the stack from both sides. The variability of the wall allow us to stipulate the exact dimensions of the required surface by printing a drawing of the graph with the desired dimensions, and using the printout as a template for constructing a wall whose undulations match those necessary for each experiment.

We ran the spatial mapping experiment on a piecewise continuous trapezoidal wall with known dimensions using

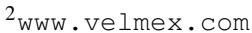

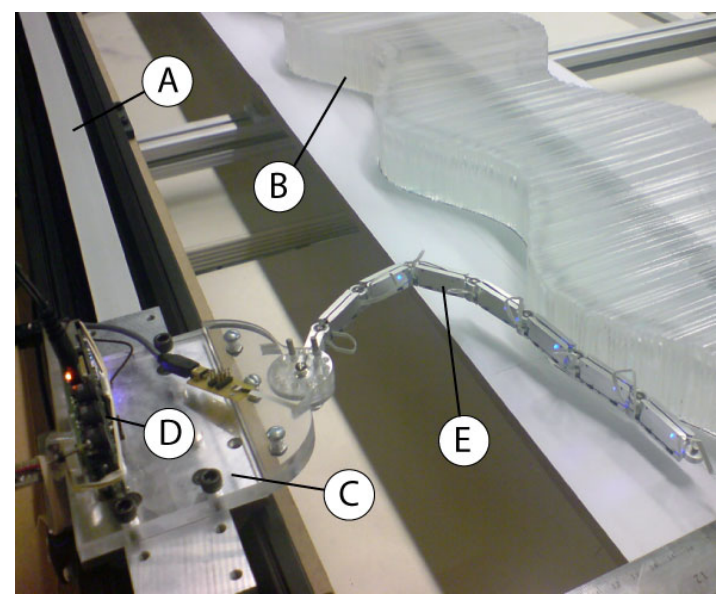

Fig. 4. A) Linear actuator, B) "Programmable" testing surface, C) Actuator car and antenna mount, D) Master computer, E) Antenna,

two different antennal stiffness profiles while keeping all other parameters constant. The first is a 3-stage, distally decreasing stiffness profile achieved by a $0.9 \mathrm{~mm}$, a $0.7 \mathrm{~mm}$ and a $0.4 \mathrm{~mm}$ diameter NiTi wire pieces fixed onto the two proximal, three middle and two distal joints respectively. The second is a constant stiffness profile with a single $0.7 \mathrm{~mm}$ diameter NiTi wire passing through all seven segments. After running the antenna at constant speed for both profiles we got the mapping results provided in Figure 3. The change in antenna posture is apparent between the two plots, where the relatively higher stiffness at the distal segments clearly prevents the inclined surfaces to be picked up by the hairs. Conversely, the relatively lower stiffness at the proximal segments causes the antenna to mostly drag behind the linear actuator car instead of keeping up which leads to some loss of accuracy towards the corners. We computed the mean spatial error of the detected contact points with the ground truth of wall as $5.0 \mathrm{~mm}$ for the decreasing stiffness and $9.4 \mathrm{~mm}$ for the constant stiffness profile.

We also performed spatial mapping experiments on a sinusoidal wall shape with spatial frequencies of $2.8 \mathrm{~m}^{-1}$, $4.2 \mathrm{~m}^{-1}, 6.3 \mathrm{~m}^{-1}, 9.5 \mathrm{~m}^{-1}$ and $14.2 \mathrm{~m}^{-1}$. The aim of this experiment was to reveal the effects of different stiffness profiles on the tactile spatial resolution. Hence we imposed the previously employed stiffness profiles-distally decreasing and constant-to our antenna. Comparing the spatial mapping results shown on figure 5 , the performance difference is clearly noticeable. Specifically, at the highest $\left(14.2 \mathrm{~m}^{-1}\right)$ wall frequency the average distance discrepancy from the ground truth for the distally decreasing stiffness is almost the same as the distance discrepancy for the constant stiffness configuration at the frequency of $6.3 \mathrm{~m}^{-1}$.

For all the experiments described above, no precurvature was imposed and the distance between the wall and the linear actuator are kept constant. For that matter non-reported experiments hint that those parameters have also significant effects on the mechanics of tactile sensing. Hence we do not claim that any of the results provided here are optimal. On 


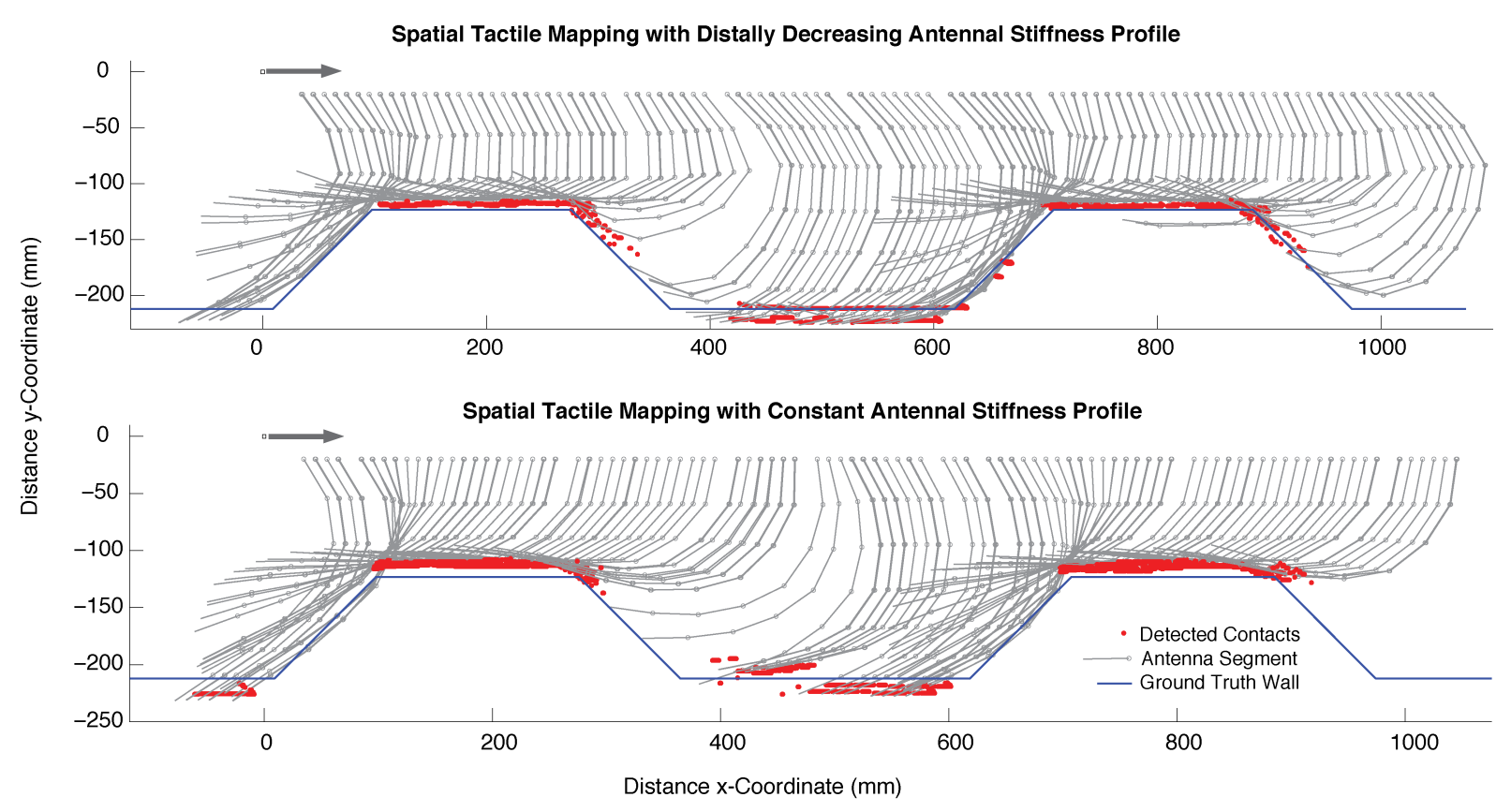

Fig. 3. Spatial mapping experiment of the antenna with 2 different stiffness profiles stiffness. The antenna sweeps the wall at $24 \mathrm{~mm} \mathrm{~s}^{-1}$. The average mapping error is computed as $10.7 \mathrm{~mm}$ from the ground truth.
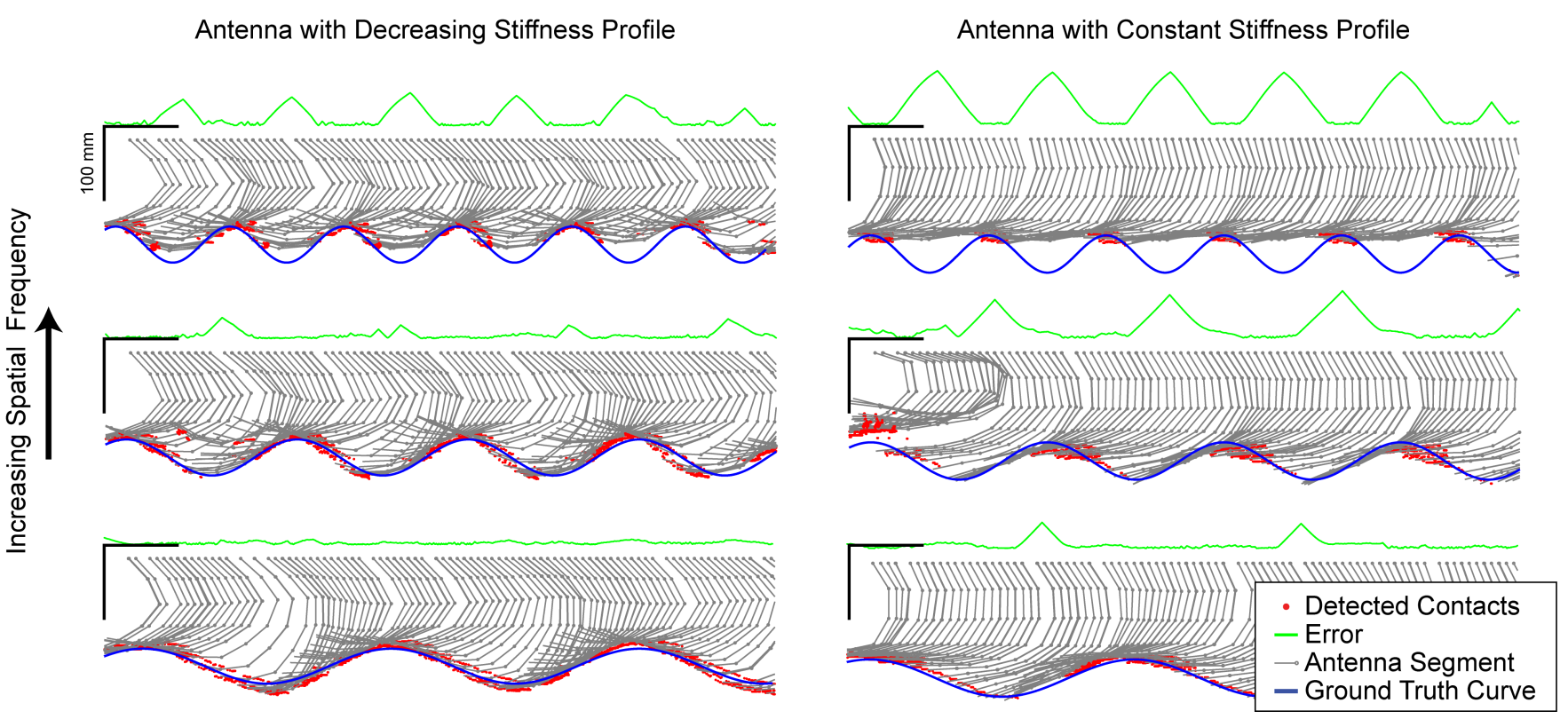

Fig. 5. Comparison of two spatial frequency experiments: antenna with a distally decreasing stiffness profile and antenna with constant stiffness profile. The frequency of the wall shape increase linearly in logarithmic scale. As the frequency increases, the wall shape cannot be captured accurately. For the antenna with decreasing stiffness, the mean spatial errors from bottom to top are: $2.7 \mathrm{~mm}, 5.1 \mathrm{~mm}, 12.1 \mathrm{~mm}$ and $25.0 \mathrm{~mm}$. For the constant stiffness antenna the mean spatial errors are (bottom to top): $5.0 \mathrm{~mm}, 14.7 \mathrm{~mm}$ and $25.9 \mathrm{~mm}$. The wall shape frequencies for adjacent plots are identical.

the contrary, we suspect that any optimal parameter value regarding antennal sensing will be highly dependent on the task or application. Yet the tunability of our antenna will allow us to explore the design space for each case.

\section{DISCUSSION}

The main design goal for the template antenna was to incorporate as many tunable design parameters as possible with the fewest number of sensors that would facilitate meaningful exploration of the mechanics antennal tactile sensing. We showed that changing mechanical properties of the antenna such as the stiffness profile affects the overall tactile sensing performance and thus prove the value of tunability. The design should also enable the antenna to adapt to different tactile sensing modalities such as wall following or shape recognition etc.

Further work for this project will continue in two parallel tracks, one for scientific research and one for engineering 
development. The first track will entail the extensive utilization of the tactile antenna to test various hypotheses regarding biological antennae. Our first milestone in that regard will be determining the mechanical contributions of distally pointing antennal spines on to the global configuration of the antenna during wall following.

Besides its scientific value, our new antenna presents new opportunities for applied robotics, especially considering the potential applications where traditional navigational instruments are insufficient or undesirable. For example sensors like sonar and vision exhibit sensitivity to surface reflectivity and lighting conditions. In particular sonar performance degrades in the presence of highly polished surfaces such as glass walls and at very close proximity; vision fails without adequate light and similarly infrared sensors-like sonarhave a minimum range below which they become unreliable. Our tests promise that bio-inspired tactile antennae can one day offer a potentially robust solution for various tasks, in which reliable robotic navigation is crucial but the limitations mentioned above may pose problems. Such areas of applications include emergency search and rescue, military operations in urban terrain, seafloor navigation and space exploration.

\section{ACKNOWLEDGMENTS}

Alican Demir designed and fabricated the antenna, and conducting experimental testing. Edward Samson helped in experimental design and testing, and in construction of the linear test facility. Noah Cowan advised on all aspects of the project. Special thanks to D. Karlsberg for help with design and fabrication of the linear actuator test facility.

\section{REFERENCES}

[1] R. J. Full and D. E. Koditschek, "Templates and anchors: neuromechanical hypotheses of legged locomotion on land," J Exp Biol, vol. 202, no. 23, pp. 3325-3332, 1999.

[2] D. A. Grimaldi and M. S. Engel, Evolution of the Insects. Cambridge University Press, May 2005.

[3] K. J. Gaston, "The magnitude of global insect species richness," Conservation Biology, vol. 5, no. 3, pp. 283-296, 1991.

[4] T. L. Erwin, "How many species are there?: Revisited," Conservation Biology, vol. 5, no. 3, pp. 330-333, 1991.

[5] R. M. May, "How many species are there on earth?" Science, vol. 241, no. 4872, pp. 1441-1449, 1988.

[6] PPS, "DigiTacts II ${ }^{\mathrm{TM}}$, tactile array sensor," Pressure Profile Systems.

[7] J. H. Solomon and M. J. Hartmann, "Biomechanics: robotic whiskers used to sense features." Nature, vol. 443, no. 7111, p. 525, Oct. 2006.

[8] R. F. Chapman, The Insects: Structure and Function, 4th ed. Cambridge University Press, November 1998.

[9] J. Okada and Y. Toh, "Antennal system in cockroaches: a biological model of active tactile sensing," International Congress Series, vol. 1269, pp. 57-60, 2004, brain-Inspired IT I. Invited papers of the 1st Meeting entitled Brain IT 2004.
[10] J. M. Camhi and E. N. Johnson, "High-frequency steering maneuvers mediated by tactile cues: antennal wall-following in the cockroach," $J$ Exp Biol, vol. 202, no. 5, pp. 631-643, 1999.

[11] J. Lee, S. N. Sponberg, O. Y. Loh, A. G. Lamperski, and N. J. Cowan, "Templates and anchors for antenna-based wall following in cockroaches and robots," IEEE Transactions on Robotics, vol. 24, no. 1, pp. 130-143, 2008.

[12] J. Okada and Y. Toh, "The role of antennal hair plates in object-guided tactile orientation of the cockroach (periplaneta americana)," Journal of Comparative Physiology A: Neuroethology, Sensory, Neural, and Behavioral Physiology, vol. 186, no. 9, pp. 849-857, 2000.

[13] B. Webb, "Can robots make good models of biological behaviour?" Behavior Brain Science, vol. 24, no. 6, pp. 1033-50; discussion 105094, Dec. 2001.

[14] T. G. Barnes, T. Q. Truong, G. G. Adams, and N. E. McGruer, "Large deflection analysis of a biomimetic lobster robot antenna due to contact and flow," Journal of Applied Mechanics, vol. 68, p. 948, 2001.

[15] N. J. Cowan, E. J. Ma, M. Cutkosky, and R. J. Full, "A biologically inspired passive antenna for steering control of a running robot," in Proceedings of the 11th international symposium on robotics research(ISRR 2003), 2003.

[16] A. G. Lamperski, O. Y. Loh, B. L. Kutscher, and N. J. Cowan, "Dynamical wall following for a wheeled robot using a passive tactile sensor," in ICRA, 2005, pp. 3838-3843.

[17] W. A. Lewinger, C. M. Harley, R. E. Ritzmann, M. S. Branicky, and R. D. Quinn, "Insect-like antennal sensing for climbing and tunneling behavior in a biologically-inspired mobile robot," in Proc. IEEE Int. Conf. Robot. Autom., 2005.

[18] D. Schneider, "Insect antennae," Annual Review of Entomology, vol. 9, no. 1, pp. 103-122, 1964.

[19] E. Staudacher, M. Gebhardt, and V. Dürr, "Antennal movements and mechanoreception: Neurobiology of active tactile sensors," in Advances in Insect Physiology, 1st ed., S. J. Simpson, Ed. Academic Press, December 2005.

[20] D. C. Sandeman, "Physical properties, sensory receptors and tactile reflexes of the antenna of the australian freshwater crayfish cherax destructor," Journal of Experimental Biology, vol. 141, no. 1, pp. 197217, 1989.

[21] J. M. Mongeau, K. Jayaram, J. Lee, N. Cowan, and R. Full, "Mechanical feedback simplifies control of cockroach antennal tactile navigation," in The Society for Integrative and Comparative Biology, Seattle, WA, 2010.

[22] R. Schafer and T. V. Sanchez, "The nature and development of sex attractant specificity in cockroaches of the genus Periplaneta. I. Sexual dimorphism in the distribution of antennal sense organs in five species," Journal of Morphology, vol. 149, no. 2, pp. 139-157, 1976.

[23] — "Antennal sensory system of the cockroach, periplaneta americana: postembryonic development and morphology of the sense organs," The Journal of Comparative Neurology, vol. 149, no. 3, 1973.

[24] G. Seelinger and T. R. Tobin, "Sense organs," in The American Cockroach, 1st ed., W. J. Bellm and K. G. Adiyodi, Eds. Springer, December 1981.

[25] A. E. Schultz, J. H. Solomon, M. A. Peshkin, and M. J. Hartmann, "Multifunctional whisker arrays for distance detection, terrain mapping, and object feature extraction," in Proc. IEEE Int. Conf. Robot. Autom., 2005.

[26] M. Lungarella, V. V. Hafner, R. Pfeifer, and H. Yokoi, "An artificial whisker sensor for robotics," in Proc. IEEE/RSJ Int. Conf. Intell. Robots Syst., Lausanne, Switzerland, 2002, pp. 2931-2936.

[27] R. A. Russell, "Using tactile whiskers to measure surface contours," in Proc. IEEE Int. Conf. Robot. Autom., Nice, France, May 1992.

[28] A. Demir, "A modular, tunable tactile antenna for exploring the mechanics of sensing," Master's thesis, Johns Hopkins University, Sept. 2009. 